\title{
Estimating gonorrhoea prevalence in young heterosexual men and women attending community-based sexual health services to inform decisions on gonorrhoea testing
}

\author{
K. TOWN ${ }^{1} *$ M. FUREGATO ${ }^{1}$, N. FIELD ${ }^{1,2}$ AND G. HUGHES ${ }^{1}$ \\ ${ }^{1}$ HIVISTI Department, National Infections Service, Public Health England, London, UK \\ ${ }^{2}$ Research Department of Infection and Population Health, University College London, London, UK
}

Received 30 August 2016; Final revision 16 December 2016; Accepted 28 December 2016; first published online 3 March 2017

\section{SUMMARY}

In England, dual tests detecting chlamydia and gonorrhoea are used in specialist and communitybased sexual health services (SHSs). Test performance is poor when prevalence is low, therefore UK national guidelines recommend against opportunistic gonorrhoea screening unless there is a clear local public health need. While surveillance data on gonorrhoea prevalence is comprehensive in specialist SHSs, it is sparse in community SHSs. We aimed to estimate gonorrhoea prevalence in heterosexual men and women aged 15-24 attending community SHSs to inform testing care pathways. We used linear and quadratic regression to model the relationship between prevalence in community and specialist SHSs in local authorities (LAs) with available surveillance data. We applied best-fitting models to predict prevalence in community SHSs in remaining LAs. Data from community SHSs were available for 102/326 LAs. There was a weak positive association between gonorrhoea prevalence in community and specialist SHSs in corresponding LAs within $\left(R^{2}=0 \cdot 13, P=0.058\right)$ and outside $\left(R^{2}=0.07, P=0.02\right)$ London.

Applying best-fitting models, we estimated a median gonorrhoea prevalence of $0.5 \%$ (mean $0.6 \%$; range $0 \cdot 2 \%-2 \cdot 7 \%$ ) in heterosexuals attending community SHSs. Despite some unexplained variation, our analyses suggest gonorrhoea prevalence in young heterosexuals attending community SHSs is below 1\% in most English LAs. Our findings re-inforce the current national guidelines that recommend care pathways for gonorrhoea testing in community SHSs include confirmatory testing to reduce the risk of misdiagnosis and inappropriate management.

Key words: Neisseria gonorrhoeae, prevalence, community, heterosexuals, England.

\section{INTRODUCTION}

Since 2008, gonorrhoea diagnoses have been increasing in England, with 41193 new cases reported in 2015 [1]. Despite this, population prevalence of gonorrhoea remains low $[2,3]$. Infection is concentrated in high-risk groups, including men who have sex with

\footnotetext{
* Author for correspondence: K. Town, HIV/STI Department, National Infections Service, Public Health England, PHE Colindale, 61 Colindale Avenue, London NW9 5EQ, UK. (Email: katy.town@phe.gov.uk)
}

men (MSM) and black Caribbeans [1]. Consequently, gonorrhoea prevalence in people attending specialist sexual health services (also known as genitourinary medicine clinics, hereafter referred to as 'specialist SHSs'), which typically serve higher risk populations, may be higher than in those attending other community-based SHSs, such as contraception clinics, pharmacies or general practice [2].

Testing for gonorrhoea in community SHSs has become widespread, in part because of the availability of dual nucleic acid amplification tests (NAATs), 
which detect Neisseria gonorrhoeae and Chlamydia trachomatis simultaneously [4]. A 2013 survey found that over half of regional local government administrative organisations in England (known as local authorities (LAs)) use dual NAATs within their chlamydia screening programmes, which aim to diagnose and control chlamydia in sexually active people aged 15-24 years $[4,5]$. However, if infection prevalence is low, so is the positive predictive value (PPV) of the test, leading to many false-positive test results. Confirmatory testing (defined as a supplementary NAAT with a different nucleic acid target) increases the PPV of the testing algorithm thereby minimising the risk of misdiagnoses and unnecessary treatment and partner notification, and in England is recommended for populations where the testing algorithm results in a PPV below 90\% [6]. Unnecessary treatment is detrimental to individual patients, both emotionally and physically, but is also problematic at the population level because unnecessary use of antibiotics may contribute to the increase of antimicrobial resistance.

Unfortunately, data on gonorrhoea prevalence in those attending community SHSs in England are sparse. The lack of local data may complicate decision-making by sexual health commissioners and service providers about gonorrhoea testing and associated care pathways in community SHSs. We used available surveillance data to develop a model estimating gonorrhoea prevalence in heterosexual men and women aged 15-24 attending community SHSs in LAs across England to inform the design of testing care pathways.

\section{METHODS}

\section{Data sources and study period}

All specialist and a minority of community SHSs in England routinely report data on gonorrhoea diagnoses among those tested to the Genitourinary Medicine Clinic Dataset (GUMCADv2) [7]. GUMCADv2 data for 2015 was used to calculate the gonorrhoea prevalence in heterosexual men and women aged 15-24 years attending specialist and community SHSs that routinely test heterosexual men and women aged 1524 for gonorrhoea (all specialist SHSs and community SHSs that conduct $\geqslant 50$ tests per year). Gonorrhoea prevalence by patient LA of residence was calculated by dividing the total number of gonorrhoea diagnoses by the total number of gonorrhoea tests.

\section{Analysis}

Linear and quadratic regression models were used to quantify the relationship between gonorrhoea prevalence in those attending specialist vs. community SHSs for LAs with data available from both service types. Model fit was assessed using the $R^{2}$ value and $t$ tests. Initial analysis suggested model-fit was improved by stratifying LAs according to patient residence within or outside London. The best-fitting models were applied to the national surveillance data (GUMCADv2) from specialist services in the remaining LAs to estimate gonorrhoea prevalence in heterosexual patients aged 15-24 years in their respective community SHSs. These estimations and the manufacturers' test sensitivity and specificity data [6] were used to calculate the PPV of the initial (reactive) gonorrhoea test result for tests conducted in community SHSs for each LA [8]. PPVs were calculated using the following equation: (sensitivity $\times$ prevalence $) /($ sensitivity $\times$ prevalence $)+(1$-specificity $) \times(1$-prevalence $))$. Analyses were completed in STATA v13.

\section{Online tool to support}

The best-fitting models developed in this analysis to predict gonorrhoea prevalence and associated PPV were converted into a user-friendly excel tool to support commissioners and service providers in making decisions about gonorrhoea testing (Supplementary material). When users select a LA, the tool presents the following local data: reported or estimated gonorrhoea prevalence, the estimated PPV of the initial (reactive) test and estimated number of false and true positives per 1000 tests performed. The tool also suggests whether or not supplementary testing is recommended to confirm infection (based on the recommended 90\% threshold outlined in the UK gonorrhoea testing guidance [6]) and presents revised estimate of the PPV following supplementary testing. The estimated PPV after supplementary testing was calculated using the PPV equation stated above, except the prevalence is calculated using the following equation: estimated number of false positives/(estimated number of false positives + estimated number of false negatives).

\section{RESULTS}

We identified 102 out of 326 LAs (29/33 in London and $73 / 293$ outside London) with data on gonorrhoea prevalence in young heterosexual men and women 
attending community SHSs. This included data from 227 specialist SHSs (33 in London and 194 outside London) and 102 community SHSs (29 in London and 73 outside London). Overall, 260499 gonorrhoea tests were reported (specialist SHSs: 112306 in London and 99339 outside London; community SHSs: 17003 in London and 31851 outside London) and 3783 gonorrhoea diagnoses were made (specialist SHSs: 1958 in London and 1388 outside London; community SHSs: 293 in London and 144 outside London).

\section{Estimated gonorrhoea prevalence in community services}

In 29 LAs in London, the median gonorrhoea prevalence was $1 \cdot 7 \%$ (mean $1 \cdot 8$, range $0.7 \%-3 \cdot 5 \%$ ) in young heterosexuals attending specialist SHSs and 1.3\% (mean $1 \cdot 5 \%$, range $0 \cdot 0 \%-4.3 \%$ ) in young heterosexuals attending community SHSs. A best-fit quadratic regression model identified a weak positive association between gonorrhoea prevalence in community and specialist SHSs in London $\left(R^{2}=0 \cdot 13, P=0 \cdot 058\right.$; Fig. 1a). Using this model, median gonorrhoea prevalence in young heterosexuals attending all community services in London was estimated to be $1 \cdot 4 \%((0 \cdot 14 \times$ specialist service prevalence $\left.{ }^{2}\right)+1 \cdot 01$; mean $1.5 \%$, range $1 \cdot 0 \%-2 \cdot 7 \%$ ).

In 73 LAs outside of London, the median gonorrhoea prevalence was $1 \cdot 1 \%$ (mean $1.3 \%$, range $0 \cdot 0 \%-5 \cdot 3 \%$ ) in young heterosexuals attending specialist SHSs and $0 \cdot 2 \%$ (mean $0.5 \%$, range $0.0 \%-6 \cdot 6 \%$ ) in young heterosexuals attending community SHSs. A best-fit linear regression model identified a positive association between gonorrhoea prevalence in community and specialist SHSs outside London $\left(R^{2}=\right.$ $0.07, P=0.02$; Fig. $1 \mathrm{~b}$ ). Using this model, median gonorrhoea prevalence in young heterosexuals attending all community services outside London was estimated to be $0 \cdot 4 \%((0.3$ specialist service prevalence $)$ $+0 \cdot 17$; mean $0 \cdot 5$, range $0 \cdot 2 \%-1 \cdot 7 \%$ ).

Overall in England, gonorrhoea prevalence among young heterosexuals tested in specialist SHSs was $\leqslant 1 \%$ in half of LAs $(160 / 326 ; 49 \cdot 0 \%)$. Gonorrhoea prevalence among young heterosexuals tested in community SHSs was estimated to be $\leqslant 1 \%$ in the majority of LAs $(285 / 326 ; 87 \cdot 4 \%)$.

\section{Estimated PPVs of reactive test results}

In London, the median gonorrhoea prevalence in young heterosexuals attending specialist SHSs was
$1.7 \%$ and estimated to be $1.4 \%$ in community SHSs. The median PPV for a reactive test result was $63.1 \%$ in specialist SHSs and was estimated to be $58.4 \%$ in community SHSs. Outside London, the median gonorrhoea prevalence was $1 \cdot 1 \%$ in specialist SHSs and estimated to be $0.4 \%$ in community SHSs. The median PPV for a reactive test result was $52.4 \%$ in specialist SHSs and was estimated to be $28.4 \%$ in community SHSs. Using these prevalence data, the estimated number of false positives and negatives and PPV estimates are presented in Table 1 .

\section{DISCUSSION}

We have developed a simple method to estimate gonorrhoea prevalence in young heterosexual men and women attending community SHSs in England, which can be used by sexual health commissioners and service providers to inform decisions about gonorrhoea testing in their local populations. A userfriendly tool based on the models developed is available online [6]. Our method is easily reproducible in other countries with high-quality surveillance data and is of value to identify populations in which the PPVs of the initial (reactive) tests will be low, leading to increased risk of misdiagnoses and unnecessary interventions.

Although our models showed significant correlations in gonorrhoea prevalence in different clinical settings within the same LA, there remained considerable unexplained variation. Predicting gonorrhoea prevalence at the LA level may mask concentrated pockets of high prevalence associated with the distribution of higher risk populations. Overlooking these clusters may result in missed opportunities for gonorrhoea testing in populations where there is a need, particularly if these groups are less likely to attend specialist SHSs. Additionally, these models do not estimate gonorrhoea prevalence in the general population, they are specific to heterosexuals populations, aged 15-24 years, attending SHSs. These patients, including those attending for chlamydia screening, are not a random sample of the population and are likely to be at higher risk of gonorrhoea infection than the general population.

Future analyses should include covariate information (currently not well completed in community SHSs) and consider modelling prevalence to a lower geographic level, such as LSOA (lower super output area). Furthermore, the models used assumed all areas outside of London are similar. However, there 
(a) London local authorities $\checkmark$ Estimate $\square$ Actual

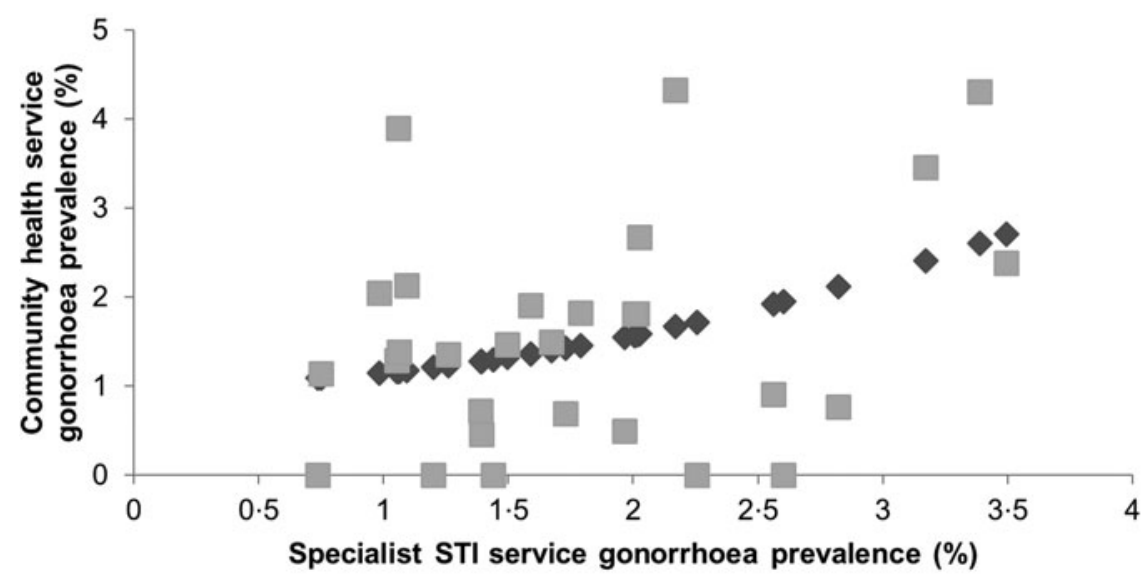

(b) Outside London local authorities

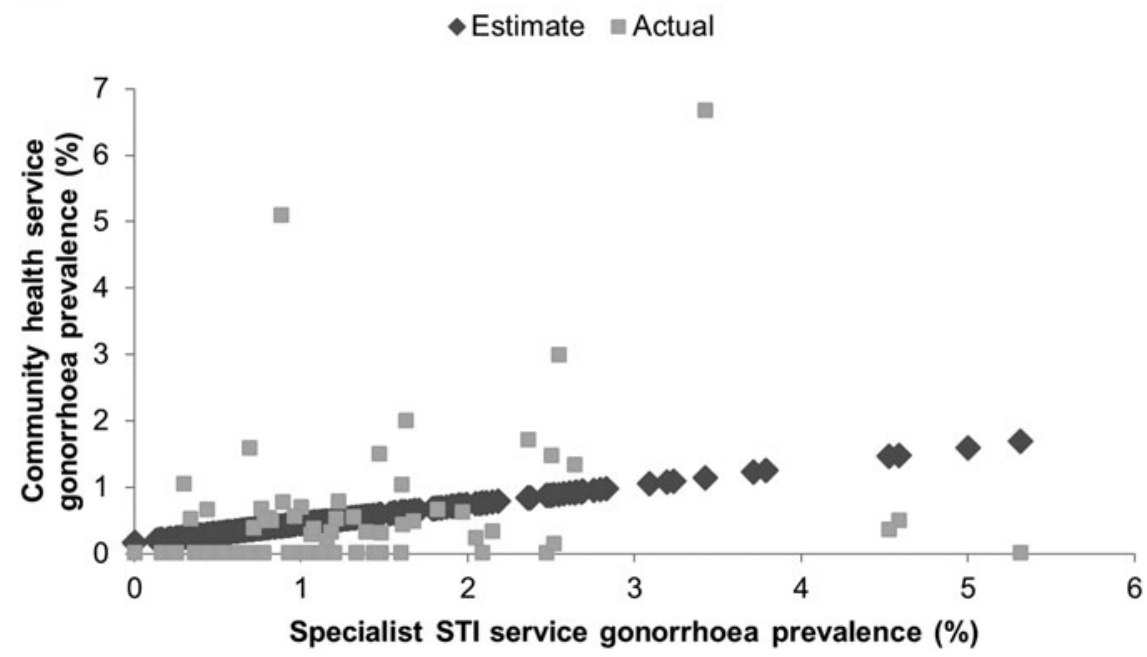

Fig. 1. Gonorrhoea prevalence in heterosexual men and women aged 15-24 years tested in specialist sexual health services (SHSs) and estimated gonorrhoea prevalence in community SHSs, data points used in model also shown. (a) London local authorities (LAs) (b) Outside London LAs.

Table 1. Estimated prevalence and associated positive predictive value (PPV), false positives and negatives after an initial (reactive) gonorrhoea test, plus the estimated PPV after supplementary testing

\begin{tabular}{llllll}
\hline \hline & $\begin{array}{l}\text { Estimated } \\
\text { prevalence }(\%)\end{array}$ & $\begin{array}{l}\text { Median PPV of } \\
\text { initial (reactive) } \\
\text { test (\%) }\end{array}$ & $\begin{array}{l}\text { Estimated number } \\
\text { of false positives } \\
\text { in } 1000 \text { tests }\end{array}$ & $\begin{array}{l}\text { Estimated number } \\
\text { of false negatives } \\
\text { in } 1000 \text { tests }\end{array}$ & $\begin{array}{l}\text { Estimated PPV } \\
\text { after supplementary } \\
\text { testing }(\%)\end{array}$ \\
\hline $\begin{array}{l}\text { London services } \\
\quad \text { Specialist }\end{array}$ & $1 \cdot 7$ & $63 \cdot 1$ & 10 & 17 & $99 \cdot 4$ \\
$\quad \begin{array}{l}\text { Community } \\
\text { Outside London services }\end{array}$ & $1 \cdot 4$ & $58 \cdot 4$ & 10 & 14 & $99 \cdot 3$ \\
$\quad \begin{array}{l}\text { Specialist } \\
\text { Community }\end{array}$ & $1 \cdot 1$ & $52 \cdot 4$ & 10 & 11 & $99 \cdot 1$ \\
\hline \hline
\end{tabular}


are distinct and important differences in service use and population demographics across and within LAs, both inside and outside of London. We have also not developed models to predict gonorrhoea prevalence in MSM because there were too few gonorrhoea tests and diagnoses reported amongst MSM attending community SHSs. This is partly due to incomplete reporting and because MSM being more likely to attend a specialist rather than community SHSs for STI (sexually transmitted infection) testing [9]. It is likely that these data would improve the precision of our model in estimating gonorrhoea prevalence within specific populations and thereby enable more targeted testing.

In this study, we assumed that reported gonorrhoea diagnoses in GUMCADv2 are confirmed diagnoses (i.e. have undergone supplementary testing). This might mean that we have overestimated gonorrhoea prevalence, and in turn overestimated the initial test PPV. However, since the prevalence is very low in most settings our principle findings are unlikely to be altered.

Our analyses suggest that gonorrhoea prevalence in heterosexual men and women attending community SHSs in England is low, especially outside London. In all community and almost all specialist SHSs, the PPV of reactive gonorrhoea test results would be below the recommended $90 \%$ threshold outlined in the UK gonorrhoea testing guidance [6]. Introducing gonorrhoea testing in community SHSs, including existing chlamydia screening programmes, may improve service provision and gonorrhoea detection in populations that do not attend specialist SHSs [10]. However, our analyses suggest that in most community services, the majority of reactive test results would be false positives.

A recent study estimating gonorrhoea prevalence in the general population, where prevalence in those aged 16-44 years was $<0 \cdot 1 \%$, showed that only 5 of 26 initial reactive test results could be confirmed, giving a PPV of $19 \%$ [3]. Likewise, data from Australia strongly suggest that an apparent rise in gonorrhoea diagnoses in women was due to an increase in falsepositive results following deployment of gonorrhoea NAATs in low prevalence populations [11]. Our study supports the principle that in all community SHSs, and, indeed, in most specialist SHSs, care pathways for gonorrhoea testing should include supplementary testing to reduce the risk of misdiagnosis, inappropriate patient management, and unnecessary treatment [6]. Our methodology and findings are likely to be relevant to many other world regions where gonorrhoea prevalence in the general population is low.

\section{SUPPLEMENTARY MATERIAL}

The supplementary material for this article can be found at https://doi.org/10.1017/S0950268817000024

\section{ACKNOWLEDGEMENTS}

No specific financial support was provided for this research. The authors thank Holly Mitchell, Helen Mebrahtu and all STI services for providing data for this analysis.

\section{DECLARATION OF INTEREST}

None.

\section{REFERENCES}

1. Public Health England. Health Protection Report. Sexually transmitted infections and chlamydia screening in England, 2015. 2016; 10: 22

2. Sonnenberg $\mathbf{P}$, et al. Prevalence, risk factors, and uptake of interventions for sexually transmitted infections in Britain: findings from the National Surveys of Sexual Attitudes and Lifestyles (Natsal). Lancet 2013; 382: 1795-1806.

3. Field $\mathbf{N}$, et $\boldsymbol{a l}$. Confirmatory assays are essential when using molecular testing for Neisseria gonorrhoeae in low-prevalence settings: insights from the third National Survey of Sexual Attitudes and Lifestyles (Natsal-3). Sexually Transmitted Infections 2015; 91: 338-341.

4. Field N, et al. Screening for gonorrhoea using samples collected through the English National Chlamydia Screening Programme and risk of false positives: a national survey of Local Authorities. BMJ Open 2014; 4: e006067.

5. Public Health England. National chlamydia screening programme standards (7th edition) (https://www.gov. uk/government/publications/ncsp-standards). Accessed 1 February 2017.

6. Public Health England. Guidance for the detection of gonorrhoea in England (https://www.gov.uk/government/publications/guidance-for-the-detection-of-gonorrhoea-in-england). Accessed 1 February 2017.

7. Savage EJ, et al. Improving surveillance of sexually transmitted infections using mandatory electronic clinical reporting: the genitourinary medicine clinic activity dataset, England, 2009 to 2013. Euro Surveillance: Bulletin Europeen sur les Maladies Transmissibles = European Communicable Disease Bulletin 2014; 19: 20981. 
8. M B. An Introduction to Medical Statistics, 3rd edn. Oxford Medical Publications, Oxford University Press, 2000.

9. Mebrahtu HFM, et al. Access of non-specialist sexual health services by men who have sex with men: do they differ from those attending specialist services? Sexually Transmitted Infections. Online First: 25 November 2016.
10. Cook PA, et al. Comparison of patients diagnosed with gonorrhoea through community screening with those self-presenting to the genitourinary medicine clinic. BMJ Open 2014; 4: e004862.

11. Chow EP, et al. Gonorrhoea notifications and nucleic acid amplification testing in a very low-prevalence Australian female population. Medical Journal of Australia 2015; 202: 321-323. 